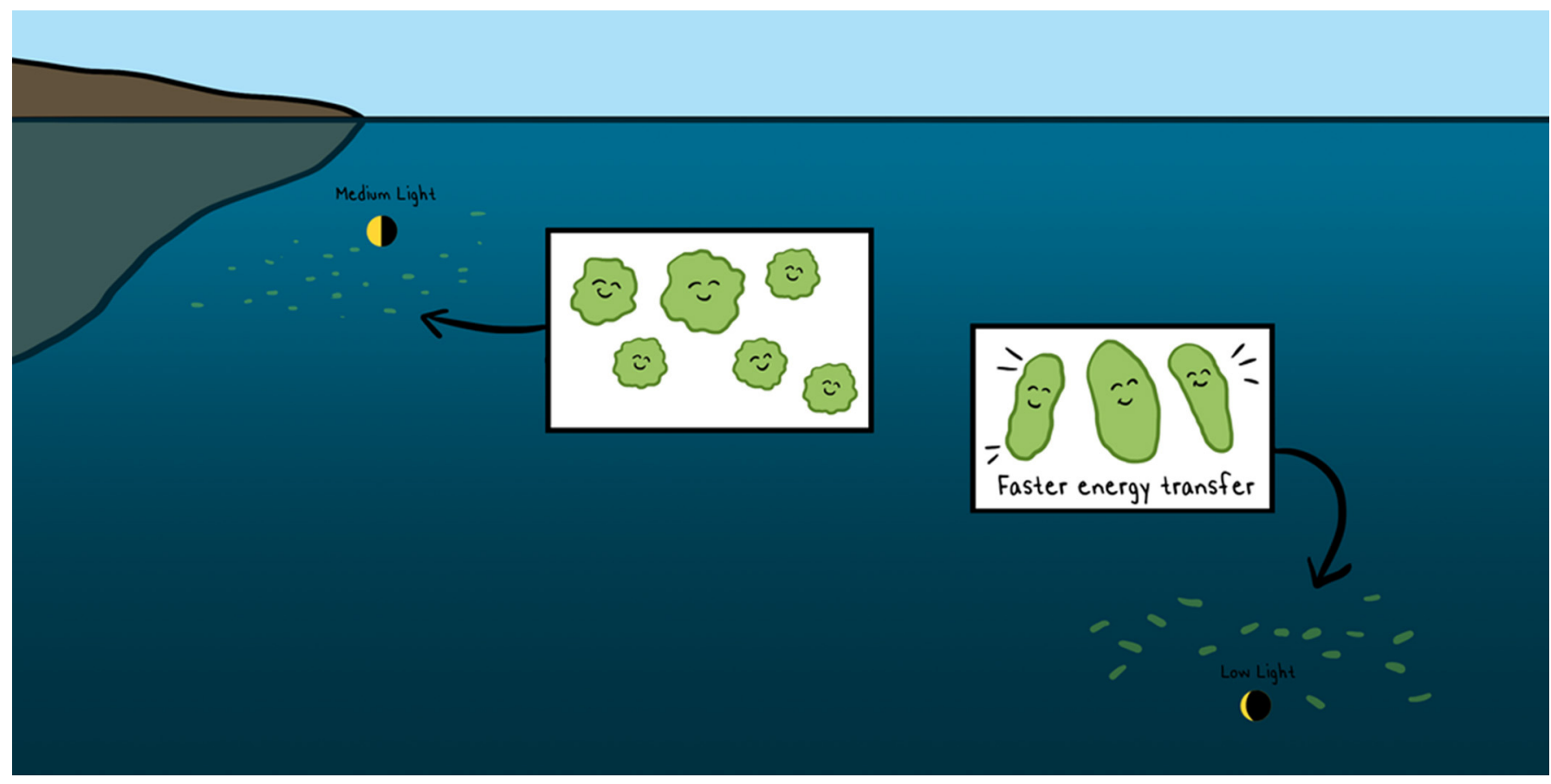

\title{
HOW DO BACTERIA PRODUCE ENERGY FROM SUNLIGHT IN THE DEEP OCEAN?
}

\section{Yuval Kolodny ${ }^{1 *}$, Yossi Paltiel $^{1}$ and Nir Keren ${ }^{2}$}

${ }^{1}$ Applied Physics Department, The Hebrew University of Jerusalem, Jerusalem, Israel

${ }^{2}$ The Alexander Silberman Institute of Life Sciences, The Hebrew University of Jerusalem, Jerusalem, Israel

YOUNG REVIEWER:

YIFTACH

AGE: 9
Photosynthesis, the process by which oxygen and sugars are created from water and carbon dioxide using the energy of the sun, is the basis of life on earth. To perform photosynthesis in changing light conditions, living creatures were required to adapt and develop sophisticated mechanisms to collect light efficiently. To understand these mechanisms, we studied the way blue-green algae (cyanobacteria), which perform photosynthesis in the sea, adapt themselves to changing light intensities, depending on the depth of the water. We found that in deep water where there is little light, the tiny antennas through which light is collected are bigger and longer. Surprisingly, although the energy is passing through a longer pathway, it actually passes faster. We found that cyanobacteria can control the energy transfer efficiency through their light-harvesting antennas by tuning the strength by which the antenna components are coupled to each other. 


\section{PHOTOSYNTHESIS}

The process used by plants, algae and certain bacteria to harness energy from sunlight and turn it into chemical energy which is stored in sugars.

\section{LIGHT ENERGY}

Light carries energy. Different light colors carry different quantity of energy. Purple is the most energetic light in the visible spectrum, while red is the least.

\section{CHLOROPHYLL}

Green pigment that composes all photosynthetic systems. It absorbs light and is a key factor in the conversion of light to chemical energy.

\section{VERTICAL MIXING}

Water column in the ocean could be stratified (i.e., each water "layer" is separated) or mixed. When an upper layer cools down it sinks, and vertical mixing occurs.

\section{CYANOBACTERIA}

A group of bacteria living in the sea and on land, which perform photosynthesis. Cyanobacteria produce $30 \%$ of the oxygen created on earth.

\section{PHOTO-}

ACCLIMATIZATION

The mechanism by which organisms match their structures and functions in response to changes in the intensity and spectrum of light around them.

\section{HOW IS PHOTOSYNTHESIS PERFORMED IN THE SEA?}

Photosynthesis is a process by which plants, algae, and bacteria build sugars from carbon dioxide and water using the energy of the sun. These sugars are the energy source for all living creatures on earth, but photosynthesis is also important for a different reason: photosynthesis releases the oxygen we breathe into the atmosphere. Photosynthesis is basically the process of transforming one type of energy (the light energy of the sun) to a different type of energy (the chemical energy stored in sugar). An important challenge in photosynthesis is the efficient capture, transfer, and transformation of light energy. To do these things, photosynthetic creatures use tiny "antennas" made from proteins, including pigment molecules that absorb the light. The energy received by the antennas is transferred through them to the places in the cell where photosynthesis occurs. There, with the help of a pigment protein called chlorophyll, photosynthesis uses the energy to build sugars.

In the sea, there is an abundance of water and carbon dioxide, but the varying amounts of available light can greatly limit photosynthesis. Light is absorbed by the water and does not penetrate down into the depths. Therefore, the deeper we go, the less light. Also, the phenomena of vertical mixing of the ocean water column occurs occasionally, so a creature found close to the surface, enjoying an abundance of light, could soon find itself in the depths, with very little light. On land, photosynthesis is usually performed by plants and trees, but in the oceans, the photosynthetic creatures are usually tiny, single-celled algae and bacteria. These creatures cannot swim, so their locations are usually determined by water currents.

Cyanobacteria (or blue-green algae) are a large group of photosynthetic bacteria abundant in the sea and on land. They are thought to have been the first creatures on earth that started performing oxygenic photosynthesis, 3-4 billion years ago [1]! Cyanobacteria have a unique structure: a large antenna to harvest light (called Phycobilisome). This antenna contains pigments, which can absorb blue light. Visible light from the sun is made up of various colors, and blue light can penetrate deeper into the water than other colors can. Cyanobacteria live at different depths, with a huge range of light available to them. Therefore, they developed complex mechanisms for dealing with changes in light intensity. Changes that occur in response to environmental conditions are called acclimatization. In the case of light, this process is called photoacclimatization.

\section{STUDYING LIGHT-HARVESTING BY CYANOBACTERIA}

We studied a species of cyanobacteria that lives throughout the water column, meaning it can adapt itself to a wide range of light intensities. 
TRANSMISSION

\section{ELECTRON}

\section{MICROSCOPE}

A powerful microscope that uses a beam of electrons, instead of light, to magnify a sample up to 10,000,000-fold.

\section{FLUORESCENT}

Having the ability to emit light after being supplied with energy. If light with a high-energy color is shined on a fluorescent material, it can emit light of a lower-energy color
These bacteria have large antennas with unique pigments, suitable for absorbing the blue light that penetrates into the deep water. We isolated these cyanobacteria from the sea and grew them in artificial seawater in the laboratory. We divided the bacteria into two transparent containers: one was illuminated with a strong blue light and the other with a weak blue light. The light intensities and color that we used correspond to the conditions we measured in the sea.

During the next 2 weeks, we tracked the changes in the cyanobacteria, using three main methods. First, using a transmission electron microscope, we looked for differences in the structures of the bacterial cells. Second, we used biochemical methods to isolate several photosynthesis-related proteins from the cells, to determine their amounts. Third, we studied the energy transfer happening in the antennas of the cyanobacteria. The pigments in the antennas are fluorescent. This means that when they are illuminated with a high-energy color of light, they emit lower energy light of a different color. For example, if the antennas are illuminated with a blue light, they may emit red or yellow light. Therefore, we could illuminate the bacteria with short flashes of light and measure the colors of light emitted, to track how quickly the light energy was traveling through the antennas.

\section{CYANOBACTERIA CHANGE TO BETTER CAPTURE LOW LEVELS OF LIGHT}

Tracking the differences between cyanobacteria grown in high and low light levels showed that bacteria grown in low light made several gradual changes, over the course of about a week, to better utilize the available light. The first change, which is visible to the naked (Figure 1 ), is the color of the cells. This means that the ratio of the different antenna pigments varied.

Using transmission electron microscopy, we saw that the cyanobacteria grown in weaker light were larger, longer, and contained several layers of membranes, typically about three for each cell (Figure 1). Cyanobacteria grown in strong light usually had a rounder shape and only one membrane layer. The membrane layers contain the cell's photosynthetic machinery. This means that a cell grown in weak light increases the number of membrane layers, to build more photosynthetic centers. This helps the cell to better utilize the limited light.

When we examined the proteins isolated from the cyanobacteria, we found that the amount of chlorophyll (the pigment of photosynthesis) was eight times higher in cyanobacteria grown in weak light compared to those grown in strong light, meaning the number of photosystems increased by a factor of eight. We also studied the antenna proteins to learn about the size of the antennas, since they are too tiny to 
Figure 1

(A) Containers in which the cells were grown, under different illumination for 10 days. They began at the same color, which gradually changed due to the change in pigment content. (B) Transmission electron microscope pictures of cyanobacteria grown in high and low levels of blue light. In low light, cells are bigger, longer, and composed of about 3 membrane layers (TM), compared to the single membrane layer usually seen in cells grown in high levels of light. The photosynthetic systems are located within the membranes, so we can deduce that the number of photosynthetic systems is much greater in low light.
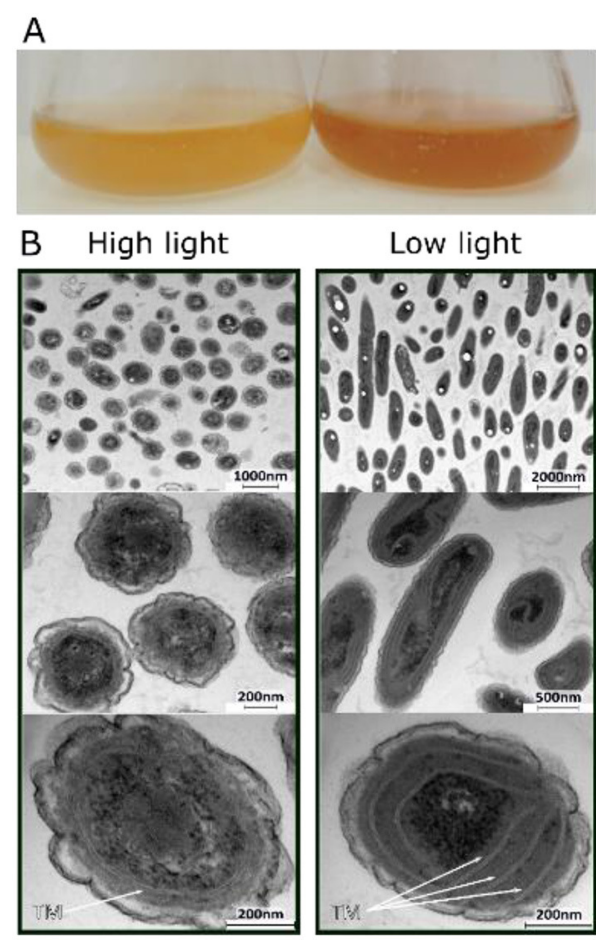

Low light

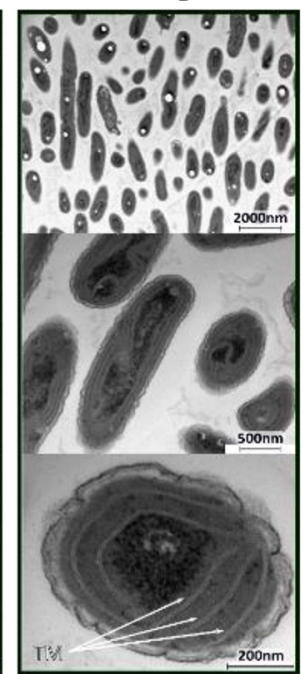

Figure 1

be seen even with our electron microscope. We calculated the ratio between the pigments that make up the antennas to chlorophyll, which is present in the photosynthetic areas. We saw that the ratio is larger in weaker light, meaning there are more pigments present in weak light. This tells us that cyanobacteria exposed to less light have larger antennas, to help them absorb as much light as possible.

The strategy of enlarging antennas to capture more light is seen in other photosynthetic organisms that must cope with a lack of light. A bigger antenna can absorb light from a bigger area, but it would seem that this would come with a disadvantage: in a larger antenna, the distance that the light energy must travel is longer. We would expect that a longer travel distance would mean it would take the light energy longer to travel through the long antenna, losing some of its energy along the way. But, to our surprise, when we measured the time it took for the light energy to move through the antenna (Figure 2), it turned out that the energy is actually transferred faster in the bacteria with bigger antennas (Figure 3)!

\section{HOW DO CYANOBACTERIA CONTROL THE LIGHT TRANSFER SPEED OF THEIR ANTENNAS?}

The surprising finding that energy transfer is actually faster in larger antennas seems to contradict the laws of classical physics. In classical physics, the light energy inside the antenna is visualized as a ball of energy that "bounces" from one pigment to another down the 
Figure 2

(A) To study how efficiently the cyanobacteria's antennas were working. we illuminated the bacteria with energetic light from a laser, and measured how long it took for the bacteria to emit a pulse of lower energy light from the pigments in their antennas. (B) Bacteria grown in weak light (green) stop emitting light more quickly than bacteria grown in strong light (pink), because the light energy is moving more quickly through their antennas to the photosynthetic systems. Since the antennas are very small, the measured time differences are in nanoseconds, meaning $1 / 1,000,000,000$ of a second.
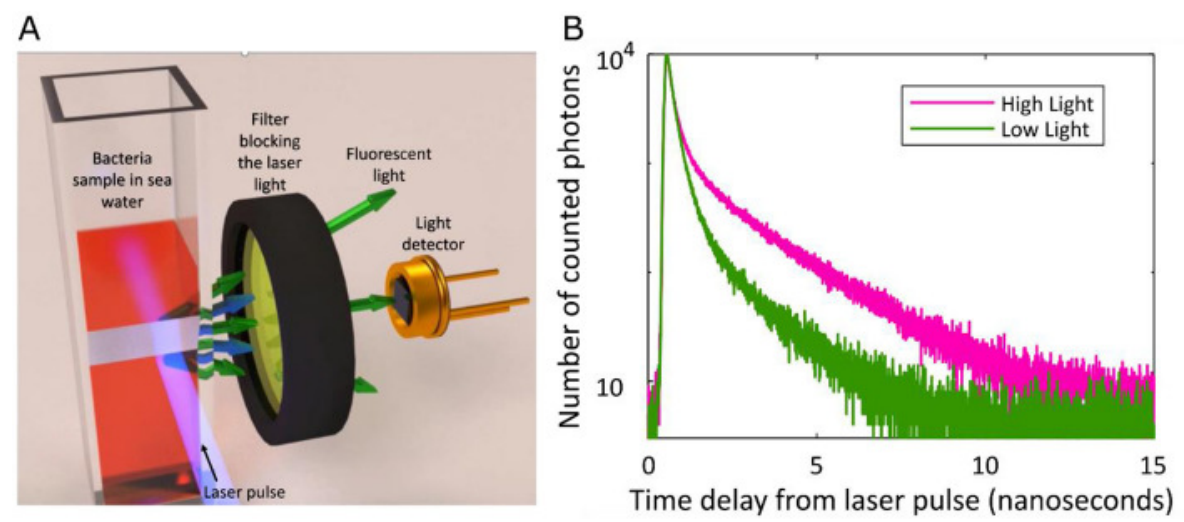

Figure 2

antenna, and in each step, the ball could roll off the path or be slowed down by obstacles. But the laws of classical physics were designed for large particles. When dealing with smaller particles like particles of light, new and intriguing laws take over, called quantum phenomena. Quantum phenomena cannot be explained using the laws of classical physics. One such quantum phenomenon is the crazy-sounding possibility that energy can be present in more than one place at the same time, more like a stretched out wave than a round ball. Energy moving as a wave can overcome the obstacles in its path with greater efficiency. To our understanding, the connections between the antenna pigments where light energy is transferred from one pigment to another are responsible for determining how the energy is transferred between pigments. This connection is on the edge between a weak coupling, for which the energy transfer follows the rules of classical physics, and a strong coupling, for which energy transfer follows quantum rules. By fine-tuning the distance between the pigments and their relative positions, cyanobacteria can switch their antennas from one state to the other! This explains why longer antennas can result in faster transfer of light energy to the photosynthetic systems.

\section{WHY IS IT IMPORTANT TO STUDY THE DETAILS OF PHOTOSYNTHESIS?}

To summarize, photosynthetic systems in cyanobacteria are very sophisticated and efficient. Cyanobacteria have evolved several impressive ways to control the efficiency of light collection, depending on the amount and color of light available to them at varying depths of the ocean.

But why is it important for scientists to understand photosynthesis in cyanobacteria? 
Figure 3

At greater depths where less sunlight penetrates,

cyanobacteria build bigger antennas to efficiently collect light energy. Although the antennas are longer, the energy transfer through them is faster. The colorful rings represent the different types of proteins that make up the antenna, each containing pigments. The blue hexagons represent the photosynthetic systems in the membrane, where the conversion of light energy into sugars is performed. Cyanobacteria can control the strength of the connections between pigment subunits, which regulates the speed that energy travels through the antennas.

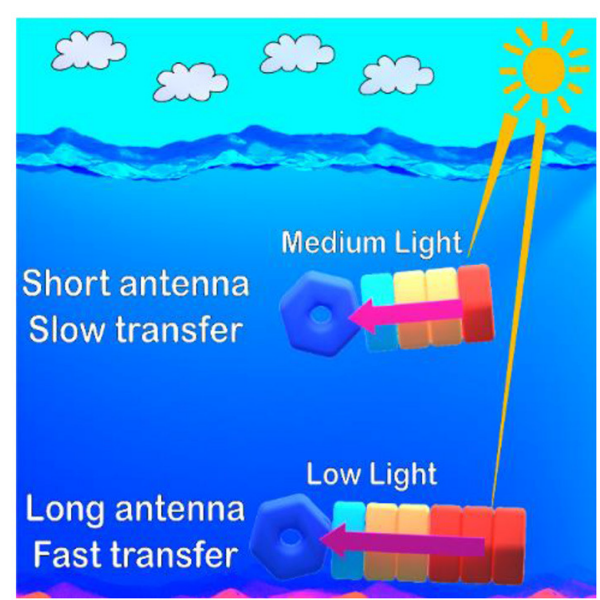

Figure 3

Photosynthesis is the fundamental process that allows life to exist on earth, and although researchers have worked for years to decipher the process, many questions remain. Often, scientists are motivated by the desire to understand processes that are fundamental to life. Beyond that basic curiosity, however, there are important practical reasons to study photosynthesis and photoacclimatization in cyanobacteria: $50 \%$ of the oxygen production on earth is performed by algae and bacteria in the ocean [2]. Global warming and other environmental changes might have dramatic influences on the oxygen production happening in our oceans, which could affect all life on earth. Understanding the details of photosynthesis might provide us with the tools and knowledge to cope with the negative effects of climate change.

Also, photosynthetic systems are incredibly efficient at transferring energy. If we can understand the processes used by these organisms, we might learn new physical principles and possibly even imitate the processes, which could result in important technological breakthroughs. For example, knowledge gained from studying the processes of photoacclimatization might aid the development of efficient solar cells. The quantum processes used by cyanobacteria might assist the development of future-generation computers that are based on quantum technologies. Full understanding of photosynthetic systems requires to combine biological knowledge with chemical, physical, and engineering knowledge. Combining knowledge from such different fields to solve big problems is possibly the greatest challenge facing the scientists of the future.

\section{ACKNOWLEDGMENTS}

Special thanks to Hagit Zer, who played a major role in the research work, and Shira Yochelis, Ido Eisenberg, and Mor Propper, for their helpful contribution and advice. 


\section{ORIGINAL SOURCE ARTICLE}

Kolodny, Y., Zer, H., Propper, M., Yochelis, S., Paltiel, Y., and Keren, N. 2020. Marine cyanobacteria tune energy transfer efficiency in their light-harvesting antennae by modifying pigment coupling. FEBS J. 288:980-94. doi: 10.1111/febs.15371

\section{REFERENCES}

1. Sánchez-Baracaldo, P., and Cardona, T. 2020. On the origin of oxygenic photosynthesis and Cyanobacteria. New Phytol. 225:1440-6. doi: 10.1111/ nph.16249

2. Field, C. B., Behrenfeld, M. J., Randerson, J. T., and Falkowski, P. 1998. Primary production of the biosphere: integrating terrestrial and oceanic components. Science 281:237-40. doi: 10.1126/science.281.5374.237

SUBMITTED: 06 May 2021; ACCEPTED: 09 June 2021;

PUBLISHED ONLINE: 30 July 2021.

EDITED BY: Idan Segev, Hebrew University of Jerusalem, Israel

CITATION: Kolodny Y, Paltiel Y and Keren N (2021) How Do Bacteria Produce Energy From Sunlight in the Deep Ocean? Front. Young Minds 9:706125. doi: 10. 3389/frym.2021.706125

CONFLICT OF INTEREST: The authors declare that the research was conducted in the absence of any commercial or financial relationships that could be construed as a potential conflict of interest.

COPYRIGHT () 2021 Kolodny, Paltiel and Keren. This is an open-access article distributed under the terms of the Creative Commons Attribution License (CC BY). The use, distribution or reproduction in other forums is permitted, provided the original author(s) and the copyright owner(s) are credited and that the original publication in this journal is cited, in accordance with accepted academic practice. No use, distribution or reproduction is permitted which does not comply with these terms.

\section{YOUNG REVIEWER}

\section{YIFTACH, AGE: 9}

My name is Yiftach, I am 9 years old and live in Israel. My favorite food is fish. My mother is a scientist and my father is an officer in the army. I am interested in biology and my hobby is gardening. We have a vegetable garden and we eat the crops that grow in it. 

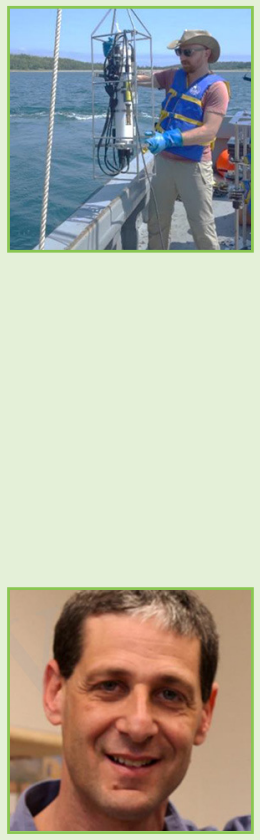

\section{AUTHORS}

\section{YUVAL KOLODNY}

Yuval Kolodny is a Ph.D. student at the Hebrew University of Jerusalem, at the Department of Applied Physics. As a kid, he did not stop asking questions about everything, all the time. He was attracted to nature and hiking, and was active in a few youth organizations. After high school, he volunteered as a youth guide for a year, and was then recruited to the Intelligence Corps. He studied electrical and computer engineering, and continued on a direct program to a Ph.D. with a specialization in nanotechnology, researching quantum biology. He also completed an MBA, and works at a startup developing medical devices. In the picture, he is lowering a device for measuring water conditions, from the deck of a research ship in the Atlantic Ocean off the shores of Canada. *yuval.kolodnyamail.huji.ac.il

\section{YOSSI PALTIEL}

Yossi Paltiel is a full professor in the Department of Applied Physics at the Hebrew University of Jerusalem, where he leads the Nano-Quantum Engineering Group. The aim of Paltiel's research is to use nanometric molecules and particles, to combine quantum physics in devices working at room-temperature. The group's specialty is nanometric devices, detectors, sources, logic, and memory devices. Prof. Paltiel is on the board of directors of the Nano Center and the Quantum Information Center of the Hebrew University. He also founded two companies: Valentis Nanotech, which uses unique features of nanocellulose to produce transparent recyclable sheets; Kiralis, dealing with the separation of chiral molecules using magnetic interactions.

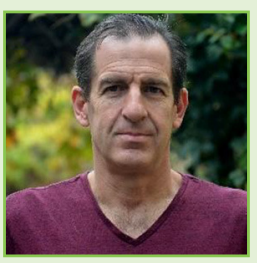

\section{NIR KEREN}

Nir Keren is a full professor in the Department of Plant Sciences and the Institute of Life Sciences at the Hebrew University. He started his scientific career chasing after lizards and snakes in the Hatseva Field School in his biotope project. Nir developed an interest in photosynthesis and did his Ph.D. under the supervision of Prof. Yitzhak Ohad in the Department of Biochemistry at the Hebrew University. After a post-doctorate at the University of Washington in Saint Lewis, he returned to the Hebrew University. His laboratory's research includes understanding how photosynthetic processes change to deal with complex environmental conditions. Nir is examining the conditions in ocean water and desert sand, trying to understand how these extreme conditions influence the photosynthesis of the microorganisms living there. 